\title{
The compact radio counterpart of IGR J20187+4041 near the flaring source AGL 2021+4029 and 3EG J2020+4017
}

\author{
Zsolt Paragi \\ JIVE, Dwingeloo, Netherlands \\ MTA Research Group for Physical Geodesy and Geodynamics, Penc, Hungary \\ E-mail: zparagi@jive.nl
}

\author{
Alfonso Trejo Cruz \\ CRyA-UNAM, Morelia, Mexico \\ E-mail: a.trejodastrosmo.unam.mx
}

\section{Elsa Giacani}

IAFE, Buenos Aires, Argentina

E-mail: egiacani@iafe.uba.ar

\section{Gloria Dubner}

IAFE, Buenos Aires, Argentina

E-mail: gdubner@iafe.uba.ar

\section{Andrei M. Bykov}

Ioffe Institute, St. Petersburg, Russia

E-mail: bykeastro.ioffe.ru

\section{Huib J. van Langevelde}

JIVE, Dwingeloo, Netherlands

Sterrewacht Leiden, Leiden University, Netherlands

E-mail: langevelde@jive.nl

We present radio results from short e-EVN (European VLBI network) observations of the counterpart to IGR J20187+4041, a hard X-ray source projected against the $\gamma$ Cygni supernova remnant (SNR). The brightest unidentified EGRET source 3EG J2020+4017 is also located in the $\gamma$ Cygni region, though its relation to IGR J20187+4041 has not been well established yet. The e-EVN observations were carried out following the AGILE detection of gamma-ray flaring activity in the region. Our observations show that the radio counterpart to the IGR source has a compact structure on the $\sim 10$ mas scales that could be related to a compact object, but no radio flaring activity has been observed. e-VLBI* is a technique which makes it possible to image the structure of radio sources at the highest angular resolution on a very short timescale.

VII Microquasar Workshop: Microquasars and Beyond

September 1-5 2008

Foca, Izmir, Turkey

*e-VLBI developments in Europe are supported by the EC DG-INFSO funded Communication Network De- 


\section{A variable gamma-ray source, and possible counterparts}

On 2008 April 28 Longo et al. (ATel \#1492) reported the detection with AGILE of a variable gamma-ray source positionally consistent with the EGRET source 3EG J2020+4017. This enigmatic object was discovered back in 1981 in the field of the $\gamma$ Cygni SNR (G78.2+2.1), and turned out to be the brightest steady-state unidentified source in the CGRO EGRET catalog (Figure 1).

In the same region Bykov et al. (2004) detected with INTEGRAL the hard X-ray source IGR J20187+4041, a likely counterpart to 3EG J2020+4017. Follow-up observations with SWIFT allowed a more precise position determination and lead to the suggestion that there is a NIR counterpart from the 2MASS catalog (2MASX J20183871+4041003, Kennea et al. 2006, ATel \#788). Based on the multi-wavelength spectrum of the source and its apparent extension in the optical and near-IR bands as well as strong absorption, it was suggested that IGR J20187+4041 is an obscured accreting object, probably an AGN at a redshift of $z<0.1$.

Recent VLA observations carried out in the C configuration at 1.4 and $5 \mathrm{GHz}$ revealed a pointlike radio source within the error box of IGR J20187+4041, positionally coincident with 2MASX J20183871+4041003 (Dubner et al., 2008 May 13, ATel \#1518). Such discovery provided further support to the obscured AGN hypothesis. Assuming the variable gamma-ray emission detected by AGILE on 2008 April 28 was due to radio-jet activity, one expects to detect this radio source on the milliarcsecond scales. Therefore, following the AGILE announcement we carried out eEVN observations to investigate this source. The e-VLBI technique, providing immediate results after the observations, is an excellent tool to conduct this kind of studies because it permits a fast organization of follow up monitoring of the target.

\section{2. e-EVN results}

We observed the radio source with the e-EVN on 2008 June 25 at $1.6 \mathrm{GHz}$. The participating radio telescopes were Cambridge, Effelsberg, Jodrell Bank (MkII), Medicina, Onsala, Torun and the phased array Westerbork. The data from the telescopes were streamed to the EVN Data Processor at JIVE real-time, for immediate processing. Because the target was very weak, we used $\mathrm{J} 2015+3710$ at a distance of 3.55 degrees for phase-referencing. Although parts of the region are very badly affected by scatter broadening by the ionized ISM in the Galactic plane, this $1 \mathrm{Jy}$ calibrator still had a correlated flux density of $400 \mathrm{mJy}$ on the longest baselines. Because this was a short exploratory project, the total on-source integration time was less than 2 hours. However, at a data rate of $512 \mathrm{Mbit} / \mathrm{s}$ this was sufficient to detect sources down to flux density levels of $\sim 100 \mu \mathrm{Jy}$.

The target is detected at the position of RA (J2000) 2018 38.7347, Dec (J2000) 4040 59.984, the position error is estimated to 2 milliarcseconds. The integrated flux density is $\sim 2.4 \mathrm{mJy}$. With a resolution of around 30 milliarcseconds, the peak brightness is $0.8 \mathrm{mJy} / \mathrm{beam}$ (Figure 2). It is hard to estimate how much scatter broadening may have affected our data from this single frequency run. But the source appears to have a compact, partially resolved structure on 10 mas scales. At $1.4 \mathrm{GHz}$ the VLA in the C-array showed a $8.7 \mathrm{mJy}$, partially resolved source, which was $2 \mathrm{mJy}$

velopments project 'EXPReS', Contract No. 02662 (http://www.expres-eu.org/). The European VLBI Network (http://www.evlbi.org/) is a joint facility of European, Chinese, South African and other radio astronomy institutes funded by their national research councils. 


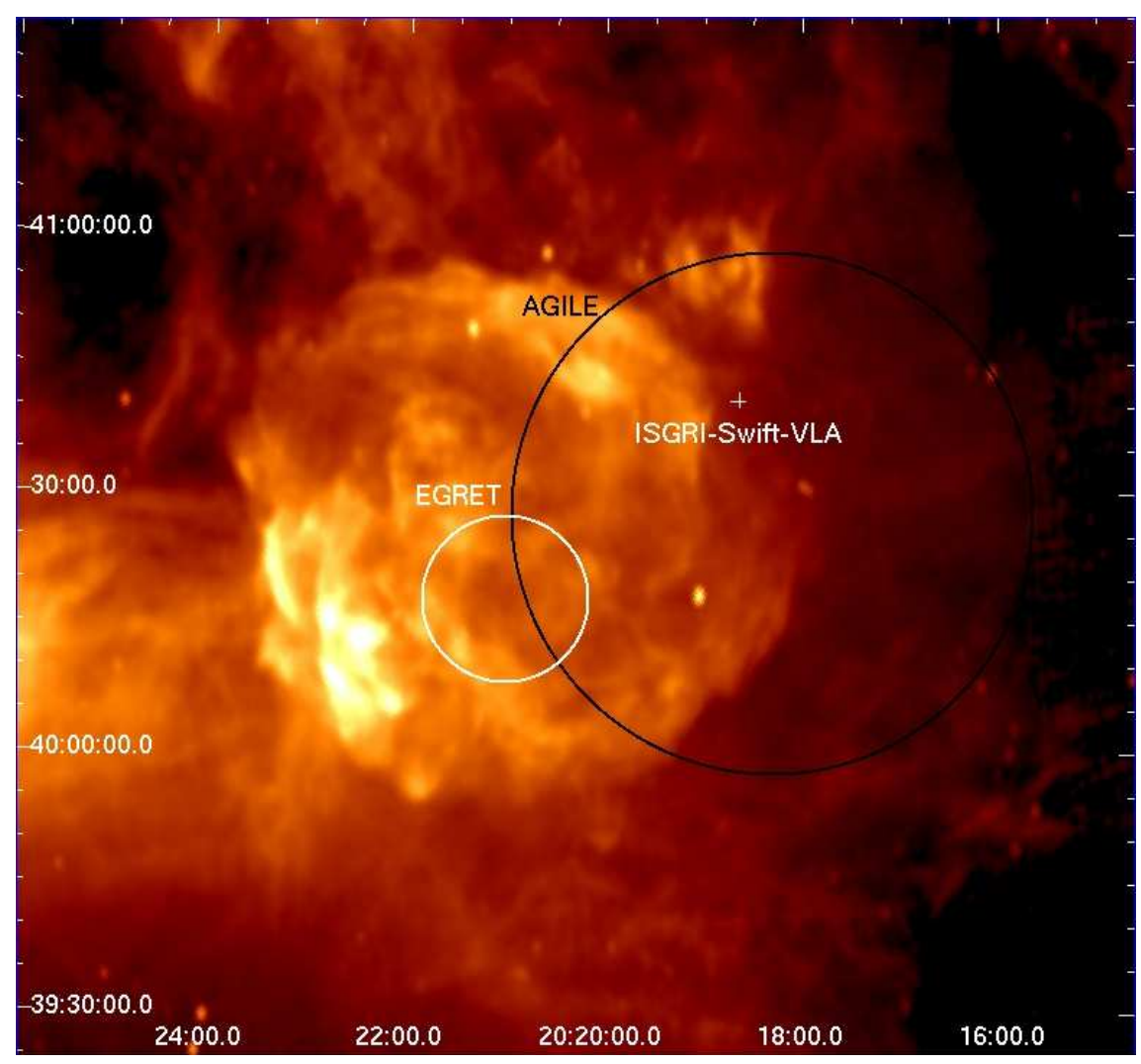

Figure 1: The $\gamma$ Cygni SNR region (image at $1.4 \mathrm{GHz}$ from a VGPS mosaic kindly provided by S. Pineault) with the positions and error circles of the unidentified EGRET source 3EG J2020+4017, the April 28 gammaflare detected by AGILE, and the hard X-ray source IGR J20187+4041 with coincident radio, IR and optical counterparts. Rebrightening of the variable gamma-source was reported in June 2008, although there was no confirmation whether this indeed agreed in position with the April 28 flare or it was an independent event.

and more point-like at $5 \mathrm{GHz}$ (Dubner et al., ATel \#1518). Comparing these with our result it was obvious that there was no strong radio flaring activity in IGR J20187+4041, and immediate further observations were not necessary. A possible interpretation of the radio structure is that we see a flat spectrum core region with the e-EVN, while at $1.4 \mathrm{GHz}$ the short baselines of the VLA picked up additional flux from extended emission, possibly a radio jet. This will be target of future multifrequency observations, which will allow us to reduce the effect of scatter broadening and better constrain the brightness temperature.

\section{The nature of the source/s}

Based on an IR spectrum of 2MASX J20183871+4041003 obtained with Keck, Goncalves et al. (ATel \#1623) accurately determined for the IR counterpart of the source a redshift $z=0.0144$ and concluded that both, redshift and X-ray properties, are consistent with a low-luminosity Seyfert nucleus. Seyfert galaxies are known to have weak, flat spectrum radio cores (e.g. Middelberg et al. 2004). However, strongly variable gamma-ray emission was never detected in these objects 


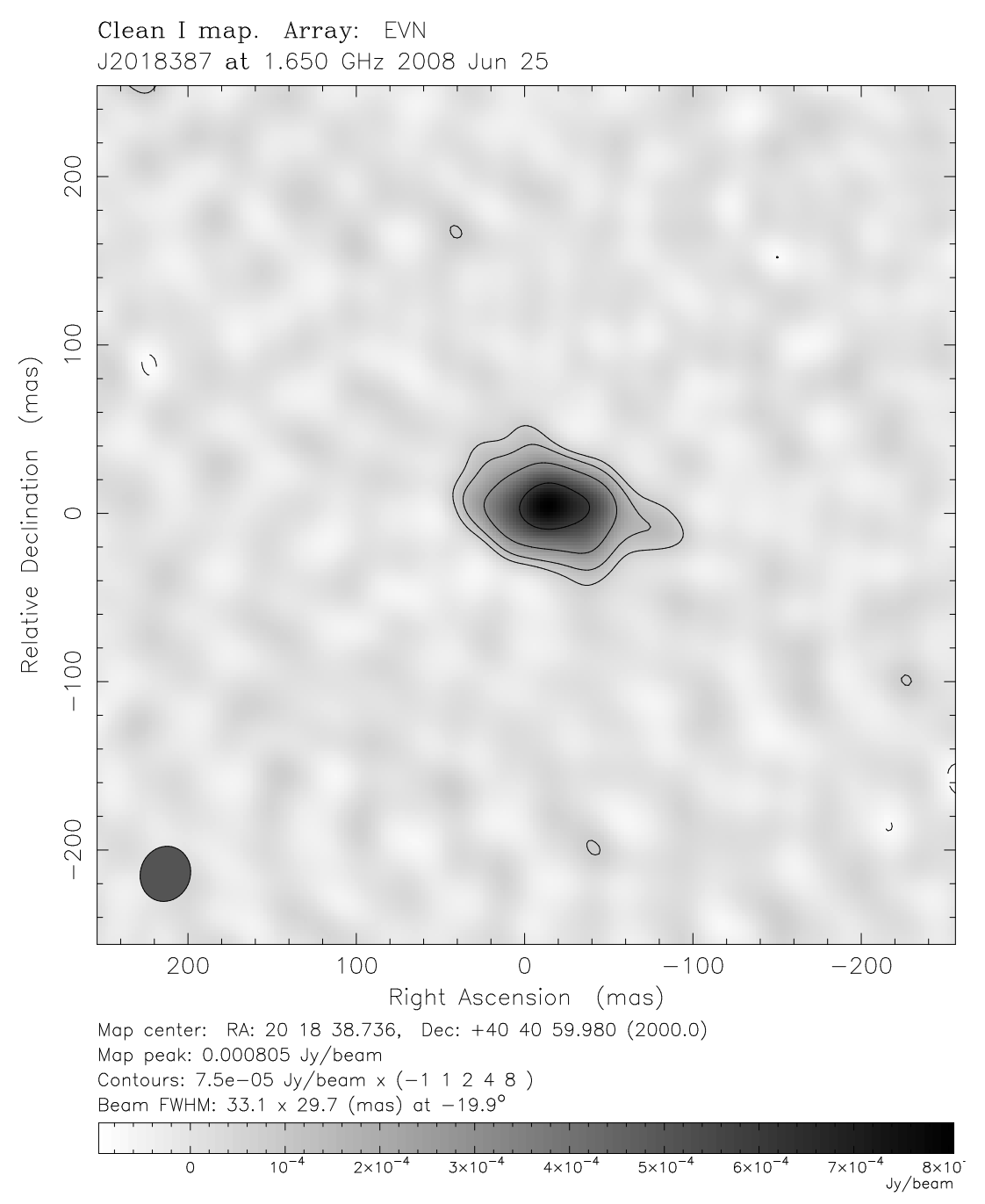

Figure 2: e-EVN detection map of the radio counterpart to IGRJ20187+4041. Note that because of the short-track observations, fine details of the image should not be overinterpreted. The source looks resolved, but the there is a possibility that scatter broadening is partially responsible for this.

before. Based on the observations available at present we are lead to the conclusion that either 2MASX J20183871+4041003/IGR J20187+4041 is a very peculiar low-luminosity AGN, or it is not related to the gamma flares detected by AGILE. Further position improvements provided by Fermi/GLAST observations will greatly help to know the true nature of the observed high-energy emission and the associated source/s.

\section{References}

[1] A. M. Bykov, Astron. Astrophys. 427, L21 (2004).

[2] E. Middelberg, A. L. Roy, N. M. Nagar, T. P. Krichbaum, R. P. Norris, A. S. Wilson, H. Falcke, E. J. M. Colbert, A. Witzel, K. J. Fricke, Astron. Astrophys. 417, 925 (2004). 